ISSN = 1980-993X-doi:10.4136/1980-993X
www.agro.unitau.br/ambi-agua
E-mail: ambi-agua@agro.unitau.br
Tel.: (12) 3625-4116

\title{
Protocolos de avaliação rápida de rios e a inserção da sociedade no monitoramento dos recursos hídricos
}

(doi:10.4136/ambi-agua.68)

\author{
Aline Sueli de Lima Rodrigues ${ }^{1}$; Guilherme Malafaia²; Paulo de Tarso Amorim \\ Castro $^{3}$ \\ Universidade Federal de Ouro Preto-MG, Rua Vereador Paulo Elias, n. 8A - Vila Itacolomy, Ouro Preto- \\ MG, CEP 35400-000, Brasil \\ E-mail: ${ }^{1}$ aline@degeo.ufop.br; ${ }^{2}$ guilherme@nupeb.ufop.br; ${ }^{3}$ paulo_de_tarso@degeo.ufop.br
}

\section{RESUMO}

A degradação dos recursos hídricos tem sido detectada e mudanças, tanto institucionais quando legislativas, têm sido requeridas. $\mathrm{O}$ uso indiscriminado dos rios provoca mudanças ecológicas, causando sérias modificações na paisagem e no regime fluvial, além de alterar a disponibilidade dos habitats e a composição trófica do ambiente aquático. Em virtude desse cenário, os cientistas têm sido pressionados a desenvolver métodos de avaliação que sejam eficientes tanto em nível da própria avaliação, quanto como auxiliares nas tomadas de decisão nos processos de gerenciamento ambiental. Nessa perspectiva, o objetivo deste trabalho é apresentar os Protocolos de Avaliação Rápida de Rios (PARs) e esclarecer as razões pelas quais esses protocolos podem promover a participação da comunidade no monitoramento dos recursos hídricos. Os PARs avaliam, de forma integrada, as características de um trecho de rio de acordo com o estado de conservação ou degradação do ambiente fluvial e suas principais características são a viabilidade econômica e a fácil aplicação. Em regiões com poucos recursos financeiros e grandes problemas de qualidade da água os PARs podem ser utilizados em programas de monitoramento ambiental. Por meio dos protocolos, a integração da comunidade no monitoramento dos recursos hídricos gera dados que representam a qualidade dos ecossistemas fluviais ao longo do tempo, sem que sejam necessários custos altos e profissionais especializados no assunto. Os PARs consistem em uma ferramenta simplificada, mas não simplista, de avaliação de rios, que incorporada aos procedimentos metodológicos adotados pelos órgãos gestores podem aproximar a sociedade civil da questão ambiental.

Palavras-chave: protocolos; rios; monitoramento dos recursos hídricos; avaliação ambiental; sociedade.

\section{Rivers rapid assessment protocols and insertion of society in monitoring of water resources}

\begin{abstract}
The degradation of water resources has been detected and changes both institutional and in the legislation have been demanded. The careless use of rivers has ecological changes as direct consequence, causing serious modifications in the landscape and fluvial regime, besides altering the availability of habitats and the trophic composition of the aquatic environment. Pressed by this scenario, scientists have been developing assessment methods that are efficient both for the evaluation itself and for supporting decision taking in the environmental management processes. In this perspective, the objective of this study is to present the Rapid
\end{abstract}


River Assessment Protocols (RAPs) and to emphasize how these protocols can promote the community participation in water resources monitoring. The RAPs can used to evaluate in an integrated form the characteristics of a river section according to the conservation or degradation condition of the fluvial environment and it is characterized by its economic viability and easy applicability. In regions with poor financial resources and serious problems of water quality, the RAPs can be used in environmental management programs. By using these protocols, the integration of the community in water resources monitoring generates data which represent the quality of fluvial ecosystems throughout time, without requesting high costs or specialized professionals. The RAPs in a simplified but not simplistic tool, which can be used in activities that aim at promoting a quick and reliable assessment of the "health" of a river.

Keywords: protocols; rivers; water resources monitoring; environmental assessment; society.

\section{INTRODUÇÃO}

Na década de 1960, ampliaram-se as preocupações com os limites do desenvolvimento, seus impactos negativos sobre a saúde humana e os níveis de degradação ambiental. Com o crescimento da população e conseqüente aumento da poluição e degradação dos corpos d’água existentes, a quantidade de água doce disponível para o uso humano e sua qualidade vêm diminuindo intensa e drasticamente (Tundisi, 2003). Segundo relatório da Organização das Nações Unidas (ONU), se medidas urgentes não forem tomadas para implementar o uso racional dos recursos hídricos, 60 países correspondendo a $75 \%$ da população mundial deverão sofrer com a falta de água no ano de 2050 (PNUD, 2003). De acordo com o Relatório de Desenvolvimento Humano $(\mathrm{RDH})$, divulgado pelo programa das Nações Unidas para o Desenvolvimento (PNUD), a falta de acesso à água e ao saneamento mata 1 criança a cada 19 segundos em decorrência de diarréia (PNUD, 2006).

Ao longo dos séculos, a ação humana tem provocado uma série de perturbações nos ecossistemas aquáticos. Historicamente, o uso das águas nunca foi realizado levando em consideração princípios de conservação (Trush et al., 2000; Barrella et al., 2001). A partir do século XVII e durante o século XVIII, observou-se o desenvolvimento da hidrologia e hidráulica, tendo como conseqüência direta o aumento dos impactos deletérios sobre os cursos d’água. No final do século XVIII, a forte intervenção da engenharia fluvial na paisagem foi observada em grandes proporções e, em meados do século XIX, a maioria dos rios europeus já tinha sido canalizada ou retificada (Saraiva, 1999; Silva et al., 2006). Nessa mesma época, iniciaram-se os estudos sobre águas interiores, contudo estes se concentravam apenas nos lagos. Em meados do século XX, os estudos passaram a ter como foco os rios, porém com uma abordagem basicamente hidrológica com fins econômicos, destacando-se a construção de barragens para a obtenção de energia elétrica, construção de eclusas e retificação de cursos para a navegação e estudos sobre saneamento em regiões criticamente poluídas. Já os estudos de abordagem ecológica surgiram logo após esse período e, atualmente, algumas pesquisas têm buscado uma abordagem global e interativa dos processos envolvidos na bacia hidrográfica (Schwarzbold, 2000).

No Brasil, o monitoramento da qualidade da água aparece na legislação federal na década de 1970, por meio da Portaria GM-0013 de 15 de janeiro de 1976 da Secretaria Especial do Meio Ambiente (SEMA), que estabeleceu uma das primeiras classificações para os corpos d'água superficiais, com os respectivos padrões de qualidade e de emissão de efluentes associados a classes de uso preponderante (Brasil, 1976). No que tange aos 
fundamentos legais da participação social no monitoramento e gestão dos recursos hídricos, nos últimos 20 anos foi possível perceber avanços importantes no setor dos recursos hídricos (Dagnino, 1994; Santos e Avritzer, 2002; Jacobi, 2003), e a mudança de uma gestão institucionalmente fragmentada para uma legislação integrada e descentralizada, aparece na edição da Lei das Águas (Brasil, 1997), considerada um dos avanços mais significativos observados nestes últimos anos.

A Lei das Águas instituiu a Política Nacional de Recursos Hídricos e criou o Sistema Nacional de Gerenciamento de Recursos Hídricos (SIGRH), incorporando os princípios estabelecidos durante a Conferência de Dublin em 1992, os quais defendem que a sociedade tem papel essencial na proteção dos ecossistemas naturais. De acordo com essa Conferência, a participação social é fundamental para o sucesso do monitoramento e gerenciamento dos recursos hídricos, pois quando a sociedade tem consciência da importância da conservação do meio ambiente, o gerenciamento é facilitado.

Além disto, há de se destacar que a Lei das Águas propõe um modelo de gestão dos recursos hídricos baseado no fortalecimento das relações entre o Poder Público e a sociedade civil, conforme disposto no capítulo I, art. $1^{\circ}$, inciso VI: "a gestão dos recursos hídricos deve ser descentralizada e contar com a participação do Poder Público, dos usuários e das comunidades”. A lei permite que o Poder Público estabeleça parceria com os usuários da água e com a sociedade civil organizada.

Bons exemplos de como podem ser estabelecidas as parcerias entre o Poder Público e a sociedade civil organizada são os Comitês de Bacias Hidrográficas (CBHs) que em 2007, contabilizaram 132 Comitês Estaduais, 7 Federais e 29 Consórcios Intermunicipais (REBOB, 2007). Os CBHs estão baseados justamente no tripé descentralização, participação e integração tendo como ênfase uma política participativa e um processo decisório aberto aos diferentes atores sociais vinculados ao uso da água, nos quais se revêem as atribuições do Estado, o papel dos usuários e o próprio uso da água. Conforme discutido por Jacobi e Barbi (2007), os atores envolvidos tendem a atuar baseados em um referencial sobre seu papel, responsabilidades e atribuições, neutralizando dessa forma, práticas predatórias orientadas pelo interesse econômico ou político. Isso facilita uma interação mais transparente e permeável no relacionamento entre os diferentes atores envolvidos - governamentais, empresariais e usuários - e limita as chances de abuso do poder (Jacobi, 2004).

Sem dúvidas, essa reorganização da gestão dos recursos hídricos substituiu práticas profundamente arraigadas de planejamento tecnocrático e autoritário. Devolve-se o poder para as instituições descentralizadas de bacia, e isso implica promover processos de negociação entre os diversos agentes públicos, usuários e sociedade civil organizada. Porém, apesar dos instrumentos legais criados, o grande desafio para o monitoramento e gestão dos recursos hídricos ainda consiste em promover uma aproximação maior entre os órgãos responsáveis pela manutenção desses recursos e a sociedade (Del Prette, 2000), embora os mecanismos de participação tenham aumentado nos últimos 10 anos (Jacobi, 2005).

Para Buss (2002), é nítido que os tomadores de decisão, as agência ambientais e as comunidades não têm agido integradamente no monitoramento dos recursos hídricos. Para o autor, esse quadro se deve não apenas à falta de implementações práticas nessas áreas, mas também à dependência de análises com tecnologias dispendiosas e que cronicamente subestimam o grau de degradação dos recursos, impedindo a percepção da realidade ambiental. Além disto, há de se ressaltar que a má articulação entre os setores governamentais e sociais, bem como a falta de diagnósticos acurados sobre as condições sócio-ambientais das bacias hidrográficas têm sido, historicamente, um grave problema. A falta de informação sobre a quantidade e a qualidade dos ecossistemas aquáticos impede a sistematização de 
dados abrangentes, o que prejudica o desenvolvimento de políticas e planejamentos de ação para os recursos hídricos (Buss, 2002).

Numa análise mais acurada, pode-se notar que várias causas podem ser apontadas para explicar essas situações, estando, de um lado, os planos político, social e econômico e, de outro, o distanciamento existente entre os conhecimentos gerados nas instituições de ensino e pesquisa e as informações que chegam às comunidades da região.

Há veemente necessidade de estabelecimento de métodos de avaliação dos cursos d'água que sejam eficientes tanto em nível da própria avaliação, como auxiliares nas tomadas de decisões nos processos de gestão ambiental e/ou como instrumento que agregue os aspectos participativos no monitoramento dos recursos hídricos. Os problemas nos dados de qualidade da água disponíveis e as deficiências das redes de monitoramento têm levado muitos pesquisadores à reavaliarem os procedimentos comumente utilizados e a pensarem no estabelecimento de métodos úteis, eficazes e confiáveis que, em conjunto com os métodos já existentes, potencializem os dados referentes ao real estado dos recursos hídricos sob avaliação, com ajuda, é claro, das comunidades locais.

Nessa perspectiva, este trabalho, por meio de uma revisão sistematizada da literatura, tem por objetivo apresentar brevemente os Protocolos de Avaliação Rápida de Rios (PARs) e, sobretudo, esclarecer as razões pelas quais esses protocolos podem promover a inserção social no processo de monitoramento e gerenciamento dos ecossistemas fluviais.

\section{METODOLOGIA}

Utilizando materiais de fonte secundária, disponíveis na Biblioteca do Instituto de Ciências Exatas e Biológicas da Universidade Federal de Ouro Preto (UFOP) e no programa de comutação bibliográfica (COMUT), bem como no banco de dados SciELO (Scientific Eletronic Library Online) e Periódicos CAPES, foram selecionados trabalhos que abordaram os PARs e a questão da participação social na gestão ambiental. A pesquisa bibliográfica incluiu artigos originais, artigos de revisão, livros, anais de congresso, editoriais e diretrizes escritos nas línguas inglesa e portuguesa. Os seguintes termos de pesquisa (palavras-chaves e delimitadores) foram utilizados em várias combinações: 1) protocolos; 2) rios; 3) participação social; 4) gestão ambiental; 5) recursos hídricos; 6) avaliação rápida; 7) sociedade; 8) monitoramento; 9) bacia hidrográfica e 10) legislação ambiental.

Para a seleção dos artigos os seguintes critérios foram utilizados: i) artigos que trataram de métodos de avaliação e monitoramento dos recursos hídricos; ii) artigos que trataram da participação da sociedade nas questões ambientais, especialmente no monitoramento dos recursos hídricos; iii) procedência e idioma: artigos nacionais e internacionais publicados em revistas especializadas; iv) tipo de publicação: periódicos e iv) ano de publicação: 1976 a 2008.

\section{RESULTADOS E DISCUSSÃO}

\subsection{Protocolos de avaliação rápida de rios}

Os Protocolos de Avaliação Rápida de Rios (PARs), nascidos de estudos liderados pela Environmental Protection Agency (EPA), durante a década de 1980, em conjunto com agências de monitoramento de águas superficiais, são utilizados para caracterizar o rio qualitativamente. É estabelecido, em princípio, um limite considerado normal baseado em locais minimamente perturbados sendo estes tomados como "referência" (Plafkin et al., 1989), partindo da premissa de que os cursos d'água pouco afetados pela ação humana 
exibem condições biológicas mais favoráveis (Minatti-Ferreira e Beaumord, 2004, 2006). O gradiente de estresse ambiental é definido a partir da observação desses locais "referência" e de locais com vários graus de alterações, desde os pouco alterados até os muito degradados.

Por definição, os PARs são ferramentas que agregam indicadores de qualidade ambiental referentes aos aspectos físicos e biológicos do ecossistema fluvial, que podem ser usados como um instrumento de avaliação dos recursos hídricos. Os PARs constituem-se em documentos de referência que reúnem procedimentos metodológicos aplicáveis à avaliação rápida, qualitativa e semi-quantitativa, de um conjunto de variáveis representativas dos principais componentes e fatores geomórficos e sedimentológicos, que condicionam e controlam os processos e funções ecológicas dos sistemas fluviais (Rodrigues e Castro, 2008; Rodrigues et al., 2008).

Em alguns países, a avaliação dos recursos hídricos vem sendo realizada por meio dos PARs, a exemplo da Austrália, onde o programa criado pelo governo e denominado Australian River Assessment System (AusRivAS), utiliza protocolos de avaliação rápida no monitoramento da qualidade ambiental dos rios do país (Parsons et al., 2002). De forma análoga, o Rapid Bioassessment Protocols (RBP's) e o River Habitat Survey (RHS), respectivamente, das agências ambientais dos Estados Unidos e do Reino Unido, adotam uma avaliação visual rápida e qualitativa para caracterizar a qualidade física global do habitat (Barbour et al., 1999).

Segundo Resh e Jackson (1993), esses protocolos são análogos aos termômetros utilizados na avaliação da saúde humana, em que valores obtidos são comparados com o que se considera "normal". As pontuações atribuídas a cada um dos parâmetros avaliados indicam o estado de "saúde" do sistema.

Para exemplificar o uso da metodologia destaca-se o estudo desenvolvido por Rodrigues (2008) no interior do Parque Estadual do Itacolomi e arredores, no município de Ouro Preto MG. Tendo em vista a importância ecológica, econômica e social dos ambientes aquáticos continentais, bem como a necessidade da inclusão de fatores abrangentes e interativos na avaliação dos recursos hídricos que visam cobrir uma ampla gama de características do rio analisado, o estudo propôs um PAR que reuniu um conjunto de parâmetros de caráter geomorfológico, sedimentológico, ecológico e biológico. A Tabela 1 sumariza os parâmetros considerados nesse protocolo.

Para cada parâmetro neste PAR, foi atribuída uma pontuação entre 0 e 20 pontos, correspondente à condição ambiental do trecho de rio sob avaliação. Os valores são distribuídos de acordo com o gradiente de estresse ambiental verificado no local da avaliação, podendo variar desde uma condição considerada "ótima" (16 a 20 pontos), até uma condição "péssima" (0 a 5 pontos), passando por situações intermediárias "boa" (11 a 15 pontos) e "regular" (6 a 10 pontos).

A pontuação aumenta na mesma proporção da qualidade do habitat e pode variar de acordo com o local das observações. Para os parâmetros cuja avaliação envolve as margens do canal (esquerda e direita), como nos parâmetros 11, 12 e 13 (apresentados na Tabela 1), a pontuação foi atribuída a cada margem separadamente. Nesses casos, as margens poderão apresentar diferentes condições ambientais e a avaliação do trecho, com relação a esses parâmetros, é obtida pelo somatório das pontuações verificadas em cada uma das margens. Notas maiores refletem um bom estado de conservação, enquanto notas menores indicam que existe um estado de degradação severa. 
Tabela 1. Relação dos parâmetros propostos no protocolo de avaliação rápida de rios proposto por Rodrigues (2008).

\begin{tabular}{l}
\hline Parâmetros \\
\hline 1. Substratos e/ou habitats disponíveis \\
2. Substratos em poços \\
3. Soterramento \\
4. Regimes de velocidade/profundidade \\
5. Diversidade dos poços \\
6. Deposição de sedimentos \\
7. Condições de escoamento do canal \\
8. Alterações no canal \\
9. Sinuosidade do canal \\
10. Freqüência de corredeiras \\
11. Estabilidade das margens \\
12. Proteção das margens pela vegetação \\
13. Estado de conservação da vegetação do entorno \\
\hline
\end{tabular}

\subsection{A participação social no monitoramento dos recursos hídricos com base em protocolos de avaliação rápida}

O primeiro ponto positivo destacado nos PARs diz respeito aos agentes sociais que podem realizar o monitoramento. Em geral, não são necessários especialistas no assunto e o método pode ser estendido a pessoas de qualquer segmento social, bastando para isso um treinamento prévio ou instruções mínimas que os permitem aplicar o protocolo sem grandes divergências. De acordo com Resh et al. (1996) e Buss (2002), os programas de monitoramento, como os que envolvem os recursos hídricos, podem ser realizados por pessoas treinadas e informadas sobre conhecimentos locais em sua região e trechos da bacia hidrográfica onde vivem, utilizando para isto metodologias padronizadas e simplificadas.

Buss et al. (2003) acreditam que um aspecto fundamental a ser considerado em um programa de monitoramento de recursos hídricos é a habilidade em traduzir as informações obtidas tanto para os gestores ambientais quanto para o público em geral. Para os autores, em muitas vezes, a complexidade dos resultados dos métodos tradicionais de avaliação impede a interpretação pelo público leigo, tornando a informação restrita e, por isso, obscura. Ao contrário, os PARs são ferramentas que permitem a formação de grupos de monitores ambientais voluntários nas comunidades, que freqüentemente podem realizar o levantamento de dados com qualidade, que podem ser considerados nos programas oficiais de monitoramento.

Um exemplo disso foi observado no estudo de Upgren (2004), no qual um PAR foi desenvolvido para monitorar os efeitos da agropecuária e das práticas de conservação dos solos na qualidade da água e do sistema fluvial, nas nascentes do rio Araguaia, GO, em que se pôde concluir que a inserção da comunidade local na avaliação ambiental dos rios da região é possível e produz resultados confiáveis. Nesse estudo, os próprios fazendeiros da região, donos de terras que os rios percorrem, foram capazes de aplicar o PAR adaptado e realizar uma avaliação periódica dos trechos, promovendo o monitoramento dos recursos hídricos da região.

No trabalho de Callisto et al. (2002), desenvolvido em trechos de bacia no Parque Nacional da Serra do Cipó - MG e no Parque Nacional da Bocaína - RJ, foi possível verificar a facilidade da aplicação de um PAR adaptado para a avaliação da diversidade de habitats nesses trechos, por estudantes voluntários. Os autores constataram que, além de não haver 
diferença significativa entre o padrão de respostas obtidas de 50 estudantes voluntários treinados e 50 não treinados, o tempo gasto na aplicação do protocolo, em cada trecho de rio analisado, foi de apenas 20 a 30 minutos, o que de acordo com os autores, reflete um bom entendimento ou uma definição clara da metodologia utilizada na avaliação rápida de habitats, ainda que em ambos os grupos tenham ocorrido uma breve explicação sobre a estrutura e funcionamento de ecossistemas lóticos.

Ainda nessa perspectiva, Rodrigues (2008) adequou um PAR para trechos de rios inseridos em campos rupestres do Estado de Minas Gerais, por meio da participação efetiva de estudantes universitários, um segmento específico da comunidade. Na ocasião, a autora ofereceu no âmbito de sua pesquisa uma oficina de monitoramento ambiental da qual participaram 42 voluntários. A idéia da incorporação da oficina de monitoramento ambiental no delineamento da pesquisa desenvolvida por Rodrigues (2008) fundamentou-se não só no envolvimento de um segmento social no monitoramento dos recursos hídricos estudados, mas também na participação deste na construção/adequação de um instrumento de avaliação holística dos sistemas fluviais.

Já Guimarães et al. (2006), no âmbito do Projeto ambiental Pampulha Limpa (PPL, 2006), criado em 2003, trabalharam com a aplicação de um PAR por centenas de crianças com idade entre 11 e 13, de escolas localizadas na bacia hidrográfica da Pampulha, Belo Horizonte, MG. O objetivo do trabalho foi justamente desenvolver nas crianças uma sensibilização, conscientização e mobilização quanto aos problemas ecológicos da Lagoa da Pampulha e a importância do monitoramento desse recurso hídrico. Na ocasião, foram realizadas saídas de campo para locais que apresentavam condições ambientais distintas, de modo a permitir comparações. Os locais visitados foram a Lagoa da Pampulha, Parque das Mangabeiras e Serra do Cipó. O "Protocolo para avaliação da saúde de rios e lagoas modificado para jovens cientistas", adaptado do proposto por Callisto et al. (2002), foi aplicado pelos escolares durante as saídas de campo. Os resultados desse trabalho mostraram que o PAR utilizado enquadra-se no elenco de ferramentas de cunho sócio-ambiental que permite comparações, suscita discussões e sensibiliza os estudantes para que adquiram valores e posturas conservacionistas, além de ter destacado a praticidade do PAR e a facilidade dos agentes envolvidos em identificar melhor o ambiente estudado por meio do protocolo.

O fato de o método contribuir com a redução de custos na avaliação dos sistemas fluviais, já que as análises físico-químicas e bacteriológicas geralmente exigem gastos onerosos, é outro aspecto positivo dos PARs. A viabilidade justifica-se, pois uma vez estabelecido o protocolo, a aplicação deste não é dispendiosa, o que permite que uma vasta malha de pontos de monitoramento seja estabelecida. Além disso, os PARs permitem a realização de uma avaliação global sobre o ecossistema investigado, conforme já discutido.

Em regiões com poucos recursos financeiros e grandes problemas de qualidade da água, por exemplo, os PARs podem ser utilizados em programas de monitoramento que envolvam a participação direta das comunidades, na tentativa de avaliar as condições básicas dos recursos hídricos em regiões onde a economia é empecilho para o desenvolvimento de projetos de monitoramento ambiental.

A integração da comunidade no monitoramento dos recursos hídricos, por meio dos PARs, gera dados que representam a qualidade dos ecossistemas fluviais ao longo do tempo, sem que sejam necessários custos altos e profissionais especializados no assunto. Esses dados podem ser úteis por detectarem possíveis interferências antrópicas sobre as fontes de água da região, além de gerarem uma consciência ambiental nas pessoas, despertando-as para a importância da manutenção dos recursos hídricos a elas disponíveis. 
Como o fortalecimento das bases sociais, no processo de gestão dos recursos hídricos (fiscalização e conservação dos recursos naturais), dar-se-á consensualmente por meio de agentes envolvidos no âmbito das bacias hidrográficas e por conteúdos metodológicos e programáticos de custos econômicos compatíveis à realidade financeira dos municípios (Garcia e Valencio, 2003; Jacobi, 2004), os dados coletados pela comunidade por meio dos PARs, podem assessorar os CBHs na condução eficaz do processo de fiscalização e gestão do patrimônio ambiental, já que os próprios agentes usuários dos recursos hídricos monitoram as condições destes.

Segundo Hannaford et al. (1997), as informações obtidas por meio dos PARs no âmbito de programas de monitoramento dos recursos hídricos, podem ser úteis para (i) sensibilizar para questões de preservação desses recursos, motivando a participação e inserção de comunidades no contexto social, político e econômico; (ii) oferecer um alerta imediato quando da ocorrência de acidentes ambientais (p.ex. derramamentos e fontes pontuais de poluição antrópica) e mortandade de peixes, contribuindo para medidas mitigadoras imediatas dos órgãos competentes e (iii) desenvolver técnicas e métodos de fácil aplicação para o desenvolvimento de programas de biomonitoramento, possibilitando a replicação da metodologia em outras sub-bacias em uma mesma região geográfica, por pessoas treinadas (não necessariamente especialistas no assunto).

Além disso, os PARs podem ser aplicados em pesquisas que exigem a obtenção de resultados rápidos, tais como em decisões de gerenciamento, podendo facilitar a transferência de conhecimento tanto para os gerenciadores quanto para a comunidade local. Podem ainda fornecer dados de vários locais para pesquisas de campo, podendo ser aplicados no desenvolvimento de metodologias em prol da conservação ambiental (Silveira, 2004).

O quadro abaixo resume de modo esquemático como os PARs podem inserir a sociedade civil nos processos de monitoramento e gestão dos recursos hídricos e aproximá-la do Poder Público (Fig. 1).

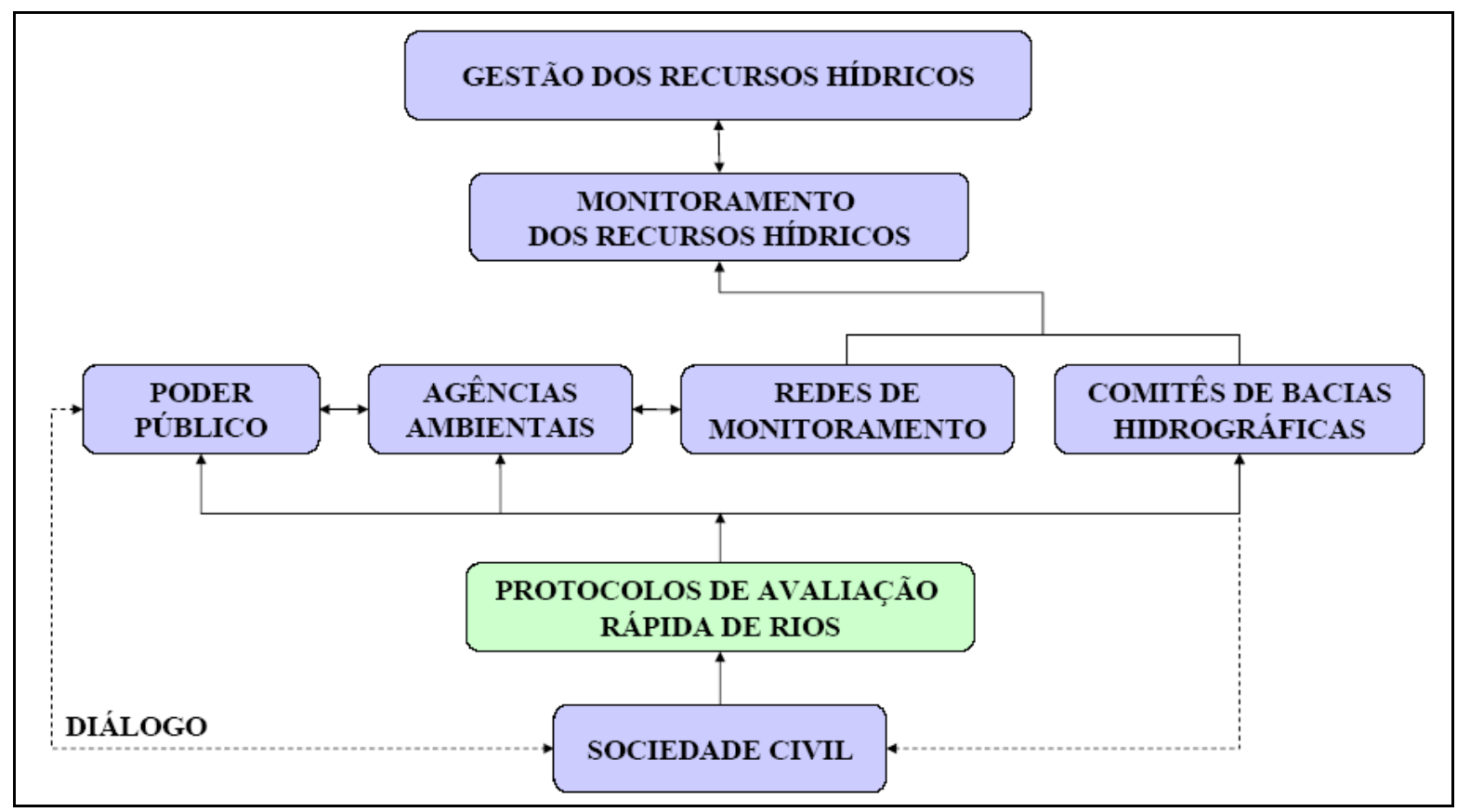

Figura 1. Fluxograma da participação social por meio dos Protocolos de Avaliação Rápida de Rios (PARs) na gestão dos recursos hídricos. 
Um exemplo de como os dados originados das aplicações dos PARs em diferentes trechos de rios poderiam contribuir para a evolução das políticas ambientais, é justamente a inclusão da metodologia proposta pelos protocolos em programas já implantados.

No Brasil, a Embrapa Meio Ambiente iniciou em 1999, um trabalho cujo objetivo é justamente incorporar a comunidade no monitoramento da qualidade da água, tendo treinado até o final do ano de 2002, cerca de 600 agentes voluntários, sendo 375 na bacia do Rio São Francisco (EMBRAPA, 2003). Em 2004, a Embrapa estruturou o Programa de Formação de Agentes Ambientais Voluntários em Monitoramento da Qualidade das Águas, o qual tem como objetivo a formação de uma rede de agentes de água que permita avaliação da qualidade dos recursos hídricos em toda a região do Submédio São Francisco. Equipamentos manuais de medição para a avaliação da qualidade da água (Ecokits) - contendo parâmetros tais como $\mathrm{pH}$, turbidez, oxigênio dissolvido, fosfato, etc. - foram sugeridos e cursos regionais de formação de agentes em monitoramento da qualidade da água foram oferecidos. Nesse caso, os PARs poderiam ser uma opção de ferramenta complementar para a avaliação dos recursos hídricos conforme acontece em países como a Alemanha (LUA, 1998) e Estados Unidos (Barbour et al., 1999).

Além disso, deve-se lembrar que os PARs podem ser utilizados pelos CBHs como instrumentos que, de fato, promovam, conforme seus princípios básicos, a participação social, principalmente por meio de programas de educação ambiental voltados para as questões dos recursos hídricos, de forma a assegurar maior participação dos usuários no próprio Comitê, a revisão do Plano de Recursos Hídricos e a efetivação de espaços à participação efetiva dos atores nos processos de gerenciamento ambiental.

\subsection{Perspectivas do uso dos protocolos de avaliação rápida de rios}

Os instrumentos legais foram e continuam sendo criados, contudo, apenas a criação de leis pouco contribui para a consolidação de uma gestão significativamente participativa, ainda mais em um país em que nem sempre o que é disposto por lei é garantido nas relações societárias. A lei sem uma ação de integração e sem uma visão positiva da participação social, pode transformar-se em um extraordinário discurso, utópico e sem aplicabilidade real.

A Lei do Voluntariado (Lei n. 9.608, de 18 de fevereiro de 1998) (Brasil, 1998) estimula o desenvolvimento de metodologias simples e eficientes para avaliação da qualidade ambiental e a transferência dessas tecnologias às comunidades. A estratégia participativa com o uso de grupos de voluntários treinados, seguindo metodologias padronizadas e de eficiência garantida, como é o caso dos PARs, é fundamental para permitir um monitoramento integrado dos sistemas aquáticos em extensas áreas, reduzindo os custos para os órgãos responsáveis pela gestão dos recursos hídricos. Além disso, proporcionam o envolvimento das comunidades locais na apropriação de ferramentas de avaliação, o que pode permitir o debate entre a comunidade e os tomadores de decisão, de forma a buscar o uso sustentável da água em todo o país.

O monitoramento dos recursos hídricos de forma efetivamente participativa requer o desenvolvimento de um processo entendível pela população, que a coloque realmente como agente participante. A implementação de programas de gerenciamento do meio ambiente por métodos que possuem uma linguagem acessível à população, pode promover o envolvimento desta com as questões ambientais e, paulatinamente, um maior interesse dessa comunidade na conservação dos recursos ambientais que a cercam.

A implementação de ferramentas acessíveis às comunidades, como os PARs, representa um grande avanço no sentido da gestão integrada da água, sua conservação e uso racional, no âmbito de uma Política Nacional de Recursos Hídricos. A demanda por dados no monitoramento dos recursos hídricos é grande, e o envolvimento das comunidades em 
projetos ambientais de monitoramento desses recursos, pode ser considerado um instrumento adicional de gestão.

É natural inferir que a utilização dos PARs nos processos de gerenciamento dos recursos hídricos representa um grande desafio a ser enfrentado pelas agências ambientais. É importante ressaltar a necessidade do desenvolvimento de um trabalho bem orientado, inovador e gerador de resultados sólidos para a gestão e o uso sustentável dos recursos hídricos, no sentido de fazer valer a utilização dos PARs como instrumentos que traduzem as reais condições dos recursos hídricos.

É importante que os PARs não sejam vistos apenas como mais um instrumento dirigido pelo Poder Público, de caráter participativo ligado aos processos de gerenciamento ambiental que venham responsabilizar a sociedade/comunidade por possíveis problemas futuros decorrentes da má avaliação ou das conseqüências dos rumos de estratégias de ação formuladas a partir dessa avaliação rápida proposta pelos PARs.

\section{CONSIDERAÇÕES FINAIS}

Baseado no que foi exposto, percebe-se que inserir a sociedade no processo de monitoramento dos recursos hídricos reflete em significativos ganhos na preservação dos ecossistemas lóticos, trazendo entre outros benefícios, a melhoria da qualidade de vida da comunidade, o desenvolvimento da economia local, o crescimento da sociedade e ainda a melhoria na qualidade de ensino. Com uma forma holística de pensar a natureza, os PARs, além de permitirem uma avaliação global do ecossistema fluvial, possibilitam a incorporação da sociedade no processo de gerenciamento do meio ambiente.

É plausível afirmar que a união entre todos os agentes envolvidos na gestão do meio ambiente pode reverter prognósticos ameaçadores e, conseqüentemente, alterar o cenário ambiental caótico em que se encontram vários locais, podendo assim, trazer esperanças sobre um futuro melhor. O método proposto consiste em uma ferramenta simplificada, mas não simplista, de avaliação de rios que, incorporada aos procedimentos metodológicos adotados pelos órgãos gestores, pode aproximar a sociedade civil da questão ambiental.

Quanto mais os atores sociais puderem conhecer, opinar e decidir sobre os problemas que os envolvem, direta ou indiretamente, vendo suas decisões serem implementadas, maior será a consolidação desses espaços, com ganhos mais amplos que extrapolarão o campo dos recursos hídricos.

\section{REFERÊNCIAS}

BARBOUR, M. T.; GERRISTSEN, J.; SNYDER, B. D.; STRIBLING, J. B. Rapid bioassessment protocols for use in streams and wadeable rivers: periphyton, benthic macroinvertebrates and fish. 2. ed. Washington: EPA 841-B-99-002, 1999. 339p.

BARRELLA, W. ; PETRERE JR., M.; SMITH, W. S.; MONTAG, L. F. A. As relações entre as matas ciliares, os rios e os peixes. In: RODRIGUES, R. R.; LEITÃO FILHO, H. (Org.). Matas Ciliares: conservação e recuperação. São Paulo: EDUSP/FAPESP, 2001, p. 187-208.

BRASIL. Ministério do Interior. Secretaria Especial do Meio Ambiente. Portaria GM/0013/15/Jan/1976: classificação das águas interiores do território nacional. Brasília: Gráfica e Editora Itamarati, 1976. 
. Lei das Águas. Lei Federal n. 9.433 de 08 de janeiro de 1997. Institui a Política Nacional de Recursos Hídricos, cria o Sistema Nacional de Gerenciamento de Recursos Hídricos, regulamenta o Inciso XIX do Artigo 21 da Constituição Federal e altera o artigo $1^{\circ}$ da Lei n. 8001 de 13 de março de 1990 que modificou a Lei n. 7990 de 28 de dezembro de 1989. Brasília, 1997.

. Lei do Voluntariado. Lei Federal n. 9.608 de 18 de fevereiro de 1998. Dispõe sobre o serviço voluntariado e dá outras providências. Brasília, 1998.

BUSS, D. F. Proteção à vida aquática, participação das comunidades e políticas de recursos hídricos. Ciência e Ambiente, v. 25, p. 71-84, 2002.

BUSS, D. F.; BAPTISTA, D. F.; NESSIMIAN, J. L. Conceptual basis for the application of biomonitoring on stream water quality programs. Cad. Saúde Pública, v. 19, n. 2, p. 465-473, 2003.

CALLISTO, M. et al. Aplicação de um protocolo de avaliação rápida da diversidade de habitats em atividades de ensino e pesquisa (MG-RJ). Acta Limnologica Brasiliensis, v. 14, n. 1, p. 91-98, 2002.

DAGNINO, E. Os movimentos sociais e a emergência de uma nova noção de cidadania. In: DAGNINO, E. (Org.). Política e sociedade no Brasil. São Paulo: Brasiliense, 1994. p.103-118.

DEL PRETTE, M. E. Apropriação de recursos hídricos e conflitos sociais: a gestão das áreas de proteção aos mananciais da região metropolitana de São Paulo. 2000. Tese (Doutorado em Geografia Humana). Faculdade de Filosofia, Letras e Ciências Humanas - Departamento de Geografia, Universidade de São Paulo, São Paulo, 2000.

EMBRAPA MEIO AMBIENTE. Elaboração de um programa de seleção, capacitação e formação de agentes de água voluntários - subprojeto 1: relatório técnico. Jaguariúna: Embrapa Meio Ambiente, 2003.

GARCIA, A. C. M. M; VALENCIO, N. F. L. S. Gestão de recursos hídricos no Estado de São Paulo: obstáculos técnicos e políticos à sustentabilidade das práticas decisórias em comitês de bacias. In: MARTINS, R.C.; VALENCIO, N.F.L.S. (Org.). Uso e gestão dos recursos hídricos no Brasil: desafios teóricos e político-institucionais. Vol. II. São Carlos: RiMa, 2003.

GUIMARÃES, A. Q.; VIANA, F. E. C.; ANDRADE, G. T. B.; VIANA, L. R. Uso de ferramentas alternativas para auxiliar saídas de campo e construção de valores conservacionistas. In: CONGRESSO IBERO-AMERICANO DE EDUCAÇÃO AMBIENTAL, 5., 2006, Joinville Anais... Brasília: MMA, 2006.HANNAFORD, M. J.; BARBOUR, M. T.; RESH, V. H. Training reduces observer variability in visual-based assessments of stream habitat. J. N. Am. Benthol. Soc., v. 16, n. 4, p. 853-860, 1997.

JACOBI, P. R. Espaços públicos e práticas participativas na gestão do meio ambiente no Brasil. Brasília: UnB, 2003.

A gestão participativa de bacias hidrográficas no Brasil e os desafios do fortalecimento dos espaços públicos colegiados. In: COELHO, V. S. P.; NOBRE, M. Participação e deliberação: teoria democrática e experiências institucionais no Brasil Contemporâneo. São Paulo: Editora 34, 2004. 
. Gestão participativa dos recursos hídricos: reflexões sobre as novas institucionalidades. São Paulo: Secretaria do Meio Ambiente de São Paulo, 2005.

JACOBI, P. R.; BARBI, F. Democracia e participação na gestão dos recursos hídricos no Brasil. Rev. Katál. Florianópolis, v. 10, n. 2, p. 237-244, 2007.

LANDESUMWELTAMT (LUA). Merkblätter nr.14. Gewässerstrukturgüte in NordrheinWestfalen Kartieranleitung (GNWK). Essen: [s.n.], 1998. p. 158

MINATTI-FERREIRA, D. D.; BEAUMORD, A. C. Avaliação rápida de integridade ambiental das sub-bacias do rio Itajaí-Mirim no Município de Brusque, SC. Health and Environmental Journal, v. 4, p. 21-27, 2004.

Adequação de um protocolo de avaliação rápida de integridade ambiental para ecossistemas de rios e riachos: Aspectos físicos. Health and Environmental Journal, v. 7, n. 1, p. 39-47, 2006.

PARSONS, M.; THOMS, M.; NORRIS, R. Australian river assessment system: AusRivAS physical assessment protocol. Monitoring river health initiative technical report number 22. Canberra: Commonwealth of Australia and University of Canberra, 2002.

PLAFKIN, J. L. et al. Rapid bioassessment protocols for use in streams and rivers: benthic macroinvertebrates and fish. Washington: U.S. Environmental Protection Agency, 1989.

PROJETO PAMPULHA LIMPA (PPL). 2006. Disponível em: $<$ http://www.pampulhalimpa.org>. Acesso em: 11 jun. 2008.

PROGRAMA DAS NAÇÕES UNIDAS PARA O DESENVOLVIMENTO (PNUD). Relatório do desenvolvimento humano 2003. Objetivos do desenvolvimento do milênio: Um pacto entre nações para eliminar a pobreza humana. Disponível em: <http://www.pnud.org.br/hdr>. Acesso em: 13 jun. 2008.

PROGRAMA DAS NAÇÕES UNIDAS PARA O DESENVOLVIMENTO (PNUD). Relatório do desenvolvimento humano 2006. A água para lá da escassez: poder, pobreza e a crise mundial da água. Disponível em: <http://www.pnud.org.br/hdr>. Acesso em: 21 jan. 2008.

REDE BRASIL DE ORGANISMOS DE BACIA (REBOB). Disponível em: <www.rebob.org.br>. Acesso em: 12 jan. 2007.

RESH, V. H.; JACKSON, J. K. Rapid assessment approaches to biomonitoring using benthic macroinvertebrates. In: ROSENBERG, D. M.; RESH, V. H. (Org.). Freshwater biomonitoring and benthic macroinvertebrates. New York: Chapman \& Hall, 1993. p. 195-233.

RESH, V. H.; MEYRS, M. J.; HANNAFORD, M. J. Macroinvertebrates as biotic indicators of Environmental Quality. In: HAUER, F. R.; LAMBERTI, G. A. (Org.). Methods in Stream Ecology. San Diego: Academic Press, 1996.

RODRIGUES, A. S. L. Adequação de um protocolo de avaliação rápida para o monitoramento e avaliação ambiental de cursos d'água inseridos em campos rupestres. 2008. 146 f. (Mestrado em Ciências Naturais) - Programa de Pós Graduação em Evolução Crustal e Recursos Naturais, Departamento de Geologia, Universidade Federal de Ouro Preto, Ouro Preto, 2008. 
RODRIGUES, A. S. L.; CASTRO, P. T. A. Protocolos de avaliação rápida: instrumentos complementares no monitoramento dos recursos hídricos. Revista Brasileira de Recursos Hídricos, v. 13, n. 1, 161-170, 2008.

RODRIGUES, A. S. L.; MALAFAIA, G.; CASTRO, P. T. A. Avaliação ambiental de trechos de rios na região de Ouro Preto-MG através de um protocolo de avaliação rápida. Revista de Estudos Ambientais, v. 10, n. 1, p. 74-83, 2008.

SANTOS, B.; AVRITZER, L. Para ampliar o cânone democrático. In: SANTOS, B.; AVRITZER, L. (Org.). Democratizar a democracia. Rio de Janeiro: Civilização Brasileira, 2002. p. 39-82.

SARAIVA, M. G. A. N. O Rio como paisagem. Gestão de corredores fluviais no quadro do ordenamento do território. Lisboa: Fundação Calouste Gulbenkian e Fundação para a Ciência e tecnologia, 1999.

SCHWARZBOLD, A. O que é um rio? Ciência \& Ambiente, v. 21, p. 57-68, 2000.

SILVA, M. et al. Futuro sustentável. Diagnóstico de Ambiente do Grande Porto, 2006. Disponível em: <http://www.futurosustentavel.org>. Acesso em: 10 abr. 2007.

SILVEIRA, M. P. Aplicação do biomonitoramento para avaliação da qualidade da água em rios. Jaguariúna: Embrapa Meio Ambiente, 2004.

TRUSH, W. J.; MCBAIN, S. M.; LEOPOLD, L. B. Attributes of an alluvial river and their relation to water policy and management. PNAS, v. 97, p. 11858- 11863, 2000.

TUNDISI, J. G. Água no século XXI: enfrentando a escassez. São Carlos: Rima, 2003.

UPGREN, A. The development of an integrated ecological assessment of the headwaters of the Araguaia River, Goiás, Brazil. 2004. Dissertação (Mestrado em Gerenciamento Ambiental, Ecossistemas e Conservação) - University of Duke, Durham, 2004. 